\title{
Reflexiones sobre las motivaciones de los necrorrománticos para la creación de productos turísticos oscuros
}

\author{
Víctor Calderón Fajardo | Dpto. de Economía y Administración de Empresas, Universidad de Málaga
}

URL de la contribución <www.iaph.es/revistaph/index.php/revistaph/article/view/5066>

La incapacidad para lograr definir qué es exactamente el turismo oscuro es lo que acarrea un caos previo de términos conceptuales similares como son Black Spots (Rojek 1993), Milking the Macabre (Dann 1994), Holocaust Tourism (Ashworth 2002), Morbid Tourism (Bloom 2000), Atrocity Tourism (Ashworth 2002), Disaster Tourism (Miller 2008), etc. Este hecho genera una falsa idea de heterogeneidad de la actividad y, posteriormente, de clasificaciones, donde resulta imposible llegar a un consenso y poder definir si se tratan de conceptos homogéneos o ramificaciones del mismo.

Sin embargo, el turismo oscuro ha llegado a definirse como una tipología muy multifacética que se interrelaciona y se reinterpreta en una variedad de contextos sociales, culturales, geográficos y políticos. Se emplea como un término inclusivo general para cualquier forma de turismo que se asocie con la muerte, con el sufrimiento, las barbaridades o los crímenes (Stone 2006). Existe desde hace siglos y es un componente participante de la historia y un instrumento para naturalizar la muerte, de hecho, se retrotrae a la Antigüedad por la fascinación por las peregrinaciones y la muerte de individuos de relevancia religiosa y mística. Aunque el primer ejemplo moderno aparece en la Inglaterra del siglo XVII donde se organizan concentraciones para contemplar las ejecuciones públicas. Igualmente, en la Francia de los siglos XVIII y XIX, donde se popularizan las visitas a las morgues en París o las escapadas para observar las muertes por guillotina (Collins-Kreiner 2016).

Por lo que hace referencia a las motivaciones de los necrorrománticos, resulta difícil de entender e identificar el porqué los turistas "actúan como actúan" (Woodside y Martin 2008). El principal problema de la motivación turística, concretamente en lo relacionado con las motivaciones del turismo oscuro, es que existe una discordancia importante e insuperable entre lo que la gente hace y lo que verdaderamente piensa, formado posiblemente por la escasa familiarización hacia nuestras propias emociones. La visita a enclaves oscuros no únicamente crea emociones positivas, sino también genera emociones negativas que influyen en la experiencia de los visitantes (Pharino, Pearce y Pryce 2018).

Por otro lado, también es pertinente subrayar que en el turismo oscuro la muerte es un aspecto inherente a la propia condición humana. Todo este planteamiento ha provocado la necesidad de desplegar mecanismos mentales para poder confrontar la idea de que, más tarde o más temprano, todos dejaremos de existir (Stone 2005). Puede, incluso, que esa misma preocupación haya sido la razón principal por la que las personas han estado vinculadas y atraídas a enclaves y eventos relacionados con el desastre, el sufrimiento, la violencia y la muerte.

Las motivaciones dependen del conocimiento, de la educación, de los vínculos e interrelaciones, de los gustos, de la intensidad y la escala de importancia y prioridades que tengan presentes los turistas. Por consiguiente, se deduce que la motivación del turista oscuro es múltiple y no necesariamente única ni discordante, puede existir un cúmulo de motivaciones que aviven el interés en la práctica del turismo oscuro. De entre todas las motivaciones, se distinguen aquellas relacionadas con los intereses históricos, los intereses culturales, el placer de la desgracia (schadenfreude ${ }^{1}$ ), el interés por lo inusual, el mero entretenimiento, el interés por rendir honores, el interés personal directo o indirecto... 


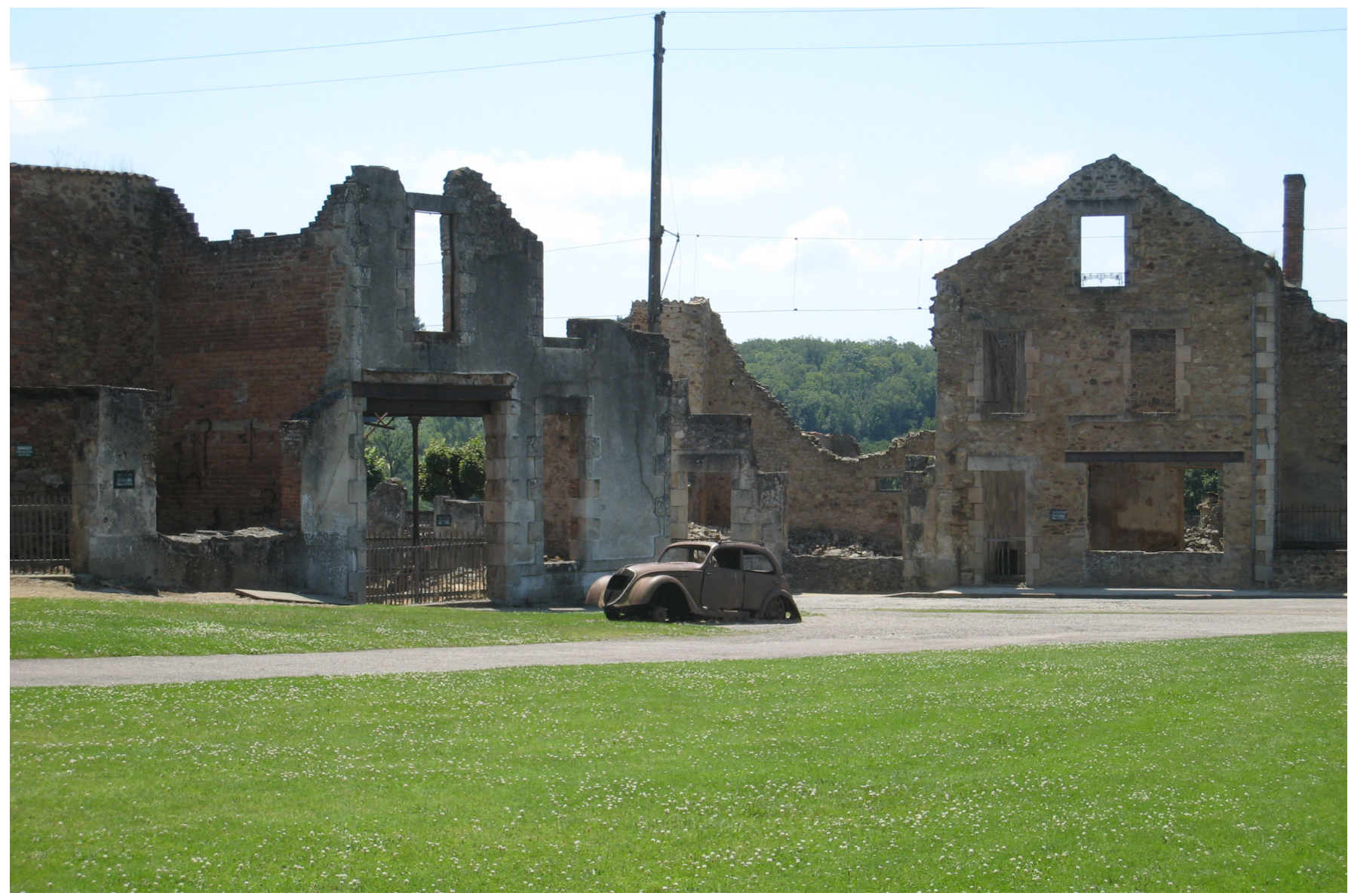

Oradour Sur Glane, ciudad conservada en el estado en el que quedó tras su destrucción el 10 de junio de 1944 por una unidad del Waffen-SS. Símbolo de los crímenes de guerra cometidos en Francia durante la ocupación nazi en la Segunda Guerra Mundial | foto IMP1

La línea divisoria de lo políticamente aceptable, moral y ético nunca ha estado clara en ninguna práctica. Resulta obvio que en algunos casos existe una aceptación, sin cuestionamientos, por parte de la sociedad, mientras que en otros se produce un rechazo draconiano. En España es un tema tremendamente espinoso debido a la herencia histórica con la que cuenta nuestro país, una guerra civil compleja, cuarenta años de dictadura y aún muchos conflictos políticos derivados del sufrimiento que han quedado pendientes y sin resolver. El turismo oscuro no ha superado completamente los prejuicios y la frontera de lo políticamente incorrecto y no se desprende íntegramente del estigma de una actividad oscura, clandestina y vergonzante que cubre una "sed mórbida" e insana.
No obstante, se aprecia que, si no hay un componente histórico-cultural claro en el turismo oscuro, siempre se estigmatiza su consumo. Es el eje que marca la línea divisoria entre lo aceptable y lo inaceptable. Otro tema importante es el marco temporal y la diversión. Cuanto más lejano en el tiempo es el recurso oscuro, mayor frivolización parece existir y menos estigma existe. Finalmente, en una dimensión ético-jurídica es interesante el matiz de "atentar contra la dignidad del ser humano", ya que resulta bastante discutible demostrar que ciertas actividades turísticas oscuras estén consideradas como un atentado o que banalizan ciertos comportamientos. El concepto ético o no ético es bastante discutible, es decir, está sujeto a múltiples interpreta- 
ciones que pueden condicionar el desarrollo de esta actividad turística, pero no hay que olvidar que las administraciones no pueden denegar la autorización para desarrollar productos turísticos oscuros por cuestionamientos éticos, subjetividad, ni arbitrariedad.

Una interpretación errónea del producto oscuro que no tiene en cuenta el componente cultural origina una ausencia total de autenticidad. Por eso, las recreaciones frívolas, subjetivas, irreales, exageradas, parciales, condicionadas a una ideología o falsas promueven el estereotipo de que el turista oscuro busca algo oscuro, y oscuro está, popularmente relacionado a algo malo y socialmente inadmisible.

Finalmente, sería tremendamente interesante plantearse desarrollar el turismo oscuro como potencial territorial con el objetivo de lograr efectos transformadores en el territorio, tales como evitar el éxodo y la despoblación, mitigar la marcada estacionalidad, la rehabilitación del patrimonio histórico y cultural o, incluso, la resurrección de áreas deprimidas (por ejemplo la "España vaciada") y un mejor despertar de letargos poscrisis (CalderónFajardo 2020). En conclusión, el turismo oscuro puede ser el catalizador de cambios capaz de dar usos a recursos endógenos latentes en tiempo convulsos, volátiles o críticos de la industria turística española.

\section{NOTAS}

1. Schadenfreude es un término de origen alemán que expresa el sentimiento de alegría o satisfacción creado por el sufrimiento, la infelicidad o la humillación de otra persona. El término análogo en español es epicaricacia.
- Bloom, T. (2000) Morbid tourism, a postmodern market niche with an example from Althorp. Norsk Geografisk Tidsskrift e Norwegian Journal of Geography, vol. 54, n. ${ }^{0}$ 1, pp. 29-36

- Calderón-Fajardo, V. (2020) Turismo dark: la muerte como nuevo negocio turístico en España. Revista Internacional de Turismo, Empresa y Territorio. RITUREM, vol. 4, n. ${ }^{\circ} 2$, pp. 1-35

- Collins-Kreiner, N. (2016) Dark tourism as/is pilgrimage. Current Issues in Tourism, 19, pp. 185-189

- Dann, G. (1994) Tourism: The Nostalgia Industry of the Future. En: Theobald, W. (ed.) Global Tourism: The Next Decade. Oxford: Butterworth Heinemannpp, pp. 55-67

- Miller, D.S. (2008) Disaster tourism and disaster landscape attractions after hurricane:An auto-ethnographic journey. International Journal of Culture, Tourism and Hospitality Research, vol. 2, n. ${ }^{\circ}$ 2, pp. 115-131

- Pharino, C., Pearce, P. y Pryce, J. (2018) Paranormal tourism: assessing tourists' onsite experiences. Tourism Management Perspectives, 28, pp. 20-28

- Rojek, C. (1993) Ways of escape: Modern transformations in leisure and travel. London: MacMillan

- Stone, P.R. (2006) A Dark Tourism Spectrum: Towards a Typology of Death and Macabre Related Tourist Sites, Attractions and Exhibitions. TOURISM: An Interdisciplinary International Journal, vol. 52, n. ${ }^{\circ} 2$, pp. 145-160

- Woodside, A.G. y Martin, D. (ed.) (2008) Tourism management: analysis, behavior and strategy. Wallingford, UK; Cambridge, USA: CAB International

\section{BIBLIOGRAFÍA}

- Ashworth, G.J. (2008) The memorialisation of violence and tragedy: Human trauma as heritage. En: Graham, B. y Howard, P. (ed.) The Ashgate companion to heritage and identity. Aldershot: Ashgate, pp. 231-244 